\title{
Synthetic Turbulence Modeling for Evaluation of Ultrasonic Cross-Correlation Flow Measurement
}

\author{
Alexander Y. Gurevich ${ }^{\mathrm{a}, *}$, Mikhail G. Goman ${ }^{\mathrm{a}}$, Yuri G. Gurevich ${ }^{\mathrm{b}}$, \\ Armando M. Lopez ${ }^{\mathrm{c}}$ \\ ${ }^{a}$ De Montfort University, Leicester, UK, LE1 9BH \\ ${ }^{b}$ Research in Flows Inc., 17 Jill Crescent, Brampton, Ontario, Canada, L6S 3 H9 \\ ${ }^{c}$ Advanced Measurement 85 Analysis Group Inc., 2396 Dunwin Drive, Mississauga, \\ Ontario, Canada, L5L $1 \mathrm{~J} 9$
}

\begin{abstract}
Performance of an ultrasonic cross-correlation flow measurement instrument may be significantly affected by turbulence at the location of the ultrasonic sensors. In this paper, a new method of generating Synthetic Turbulence is presented, to provide an effective tool for creating a variety of turbulent fields, which can be used to model and analyze instrument performance under different flow conditions. In the proposed method, a turbulent field is presented as a Fourier time-series in each point in space. Turbulence structures are defined by a spatial distribution of phase functions for each harmonic. Principles of designing a phase function to achieve the desirable distribution of turbulence scales, and two-point correlations, are outlined by considering the example of Uniform Isotropic Turbulence. One application of this method, presented in this work, is the mathematical modeling of ultrasonic cross-correlation flow measurement. Results predicted by the proposed mathematical model show good agreement with experimental data.
\end{abstract}

Keywords: synthetic turbulence, turbulence model, ultrasonic flow measurement, cross-correlation flow meter, non-intrusive flow measurement

\footnotetext{
*Corresponding author, current address: Advanced Measurement \& Analysis Group Inc., 2396 Dunwin Drive, Mississauga, Ontario, Canada, L5L 1J9

Email addresses: alexander.gurevich@email.dmu.ac.uk (Alexander Y. Gurevich), mgoman@dmu.ac.uk (Mikhail G. Goman), yuri.gurevich@rif-inc.ca (Yuri

G. Gurevich), alopez@amag-inc.com (Armando M. Lopez)
} 


\section{Introduction}

Ultrasonic cross-correlation flow measurement instrumentation may be significantly affected by turbulence characteristics at the location of the sensors. Demand for reducing flow measurement uncertainty, creates and maintains interest in evaluation of the effect of turbulence on flow meter performance $[1,2,3,4,5]$. A synthesized, turbulent-like field, called Synthetic Turbulence, can be used to model and analyze flow measurement instrument performance under different conditions. Methods of generating Synthetic Turbulence have been used for the last three decades, mainly to provide inlet boundary conditions for Direct Numerical Simulation (DNS) and for Large Eddy Simulation (LES). The main requirement in such an application is to reduce the transitional area affected by the boundary conditions. Detailed discussions of this problem are presented in $[6,7,8,9,10,11,12,13]$. Different Synthetic Turbulence models have also been developed for a number of specific applications, such as development of sub-grid turbulence models for LES [14], and for many others.

The objective of the present work, is to develop a method of generating a variety of turbulent fields for modeling the turbulence effect on flow measurement instruments. In this application, the dimensions of the flow domain of interest are small. It is defined by the size of the flow sensor, which usually is much smaller than the size of the domain where flow characteristics are formed. Often, it is the same order of magnitude of the spatial scale of turbulence, or smaller.

The most common method of generating synthetic turbulence is the spectral method, where the turbulence is presented as a Fourier series of temporal and spatial harmonics with random factors $[11,12,13]$. Another approach, the superposition method, is based on creating a family of functions representative of a turbulent structure, and superimposing such functions onto the same domain, creating a turbulent field [6, 7]. An extension of this approach, is the fractal method used to describe small scale turbulence for sub-grid models in LES [14]. The filtering method is another approach where desirable spatial structures are obtained by applying specially designed filters to a random digital distribution [11].

The method presented in this paper can be classified as a combination of the spectral and superposition methods. It is based on the presentation of a turbulent field as a Fourier series of time harmonics, where the amplitude and phase at each harmonic is a function of spatial variables. In contrast to exist- 
ing methods, the phase functions are not necessary linear, but are designed to obtain the desirable turbulent field. Examples of generating a turbulent velocity field in a pipe with desired behavior of two-point correlations are presented. Results are compared with experimental data.

An application of the presented modeling method, is the mathematical modeling of ultrasonic cross-correlation flow measurement. The principal of cross-correlation flow measurement is as follows: propagation of the turbulence field along the pipe is detected by ultrasonic beams transmitted through the flow medium, and these received ultrasonic signals are used to derive flow characteristics.

The principles of operation of a cross-correlation flow meter were first described in [16] mainly for multi-phase flows. The first attempts to develop a mathematical model of this instrument were made using the concept of a tracer $[17,18]$ without consideration of the specific mechanism of the effect of the tracer on the ultrasonic beam. The first analysis of the effect of turbulent flow on an ultrasonic beam in a cross-correlation flow measurement for a single phase flow was presented in [19], where a direct relation between perturbations of the phase of the ultrasonic wave, and the turbulent velocity, was obtained. This relation was used to develop a simple semi-empirical model of cross-correlation flow measurement in developed pipe flow. A more comprehensive model for a single phase flow was developed [20, 21], using the same relation between the phase of the ultrasonic beam and the turbulent velocity. Turbulence characteristics required by the model were obtained by solving the Reynolds Equations using the $k-\epsilon$ turbulence model, and using the approximation of isotropic uniform turbulence.

The mathematical model presented in this paper deals directly with the turbulent velocity field, and does not use the approximation of isotropic uniform turbulence. Results predicted by the mathematical modeling were compared with experimental data obtained in the Utah Water Research Laboratory in Utah State University, using the ultrasonic non-intrusive crosscorrelation flow meter CROSSFLOW, developed by AMAG Inc, Canada (www.amag-inc.com). 
An installation of the CROSSFLOW meter on a feedwater pipe of a nuclear power plant is shown in Fig. 1 (top left image). The CROSSFLOW high temperature ultrasonic transducer is shown in Fig. 1 (top right image). Two signals, representing turbulent perturbation of upstream (A, yellow) and downstream (B, red) ultrasonic beams, are shown in Fig. 1 (bottom left plot). The cross-correlation function of these signals is shown in Fig. 1 (bottom right plot).
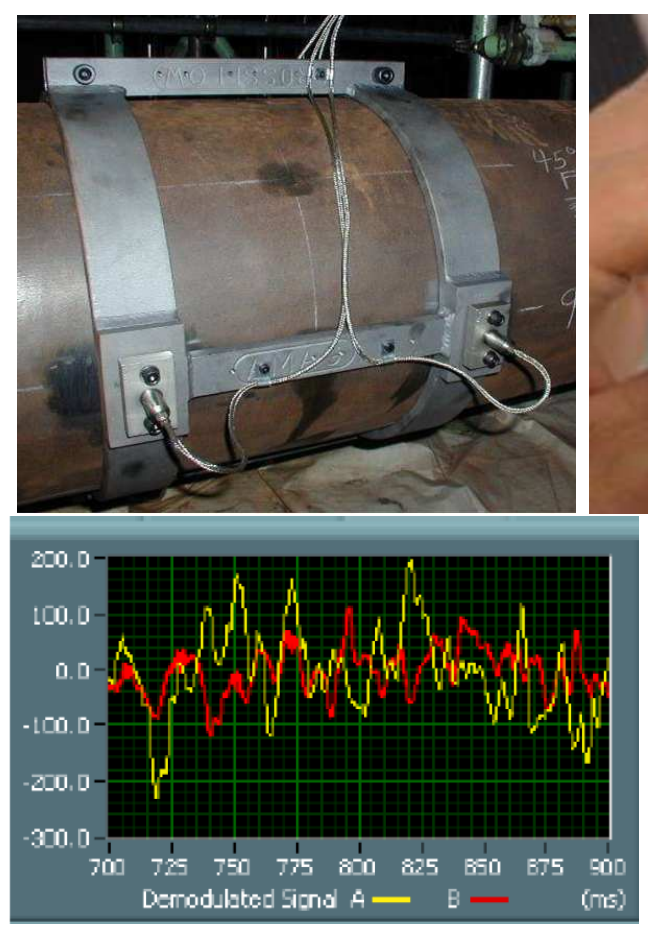
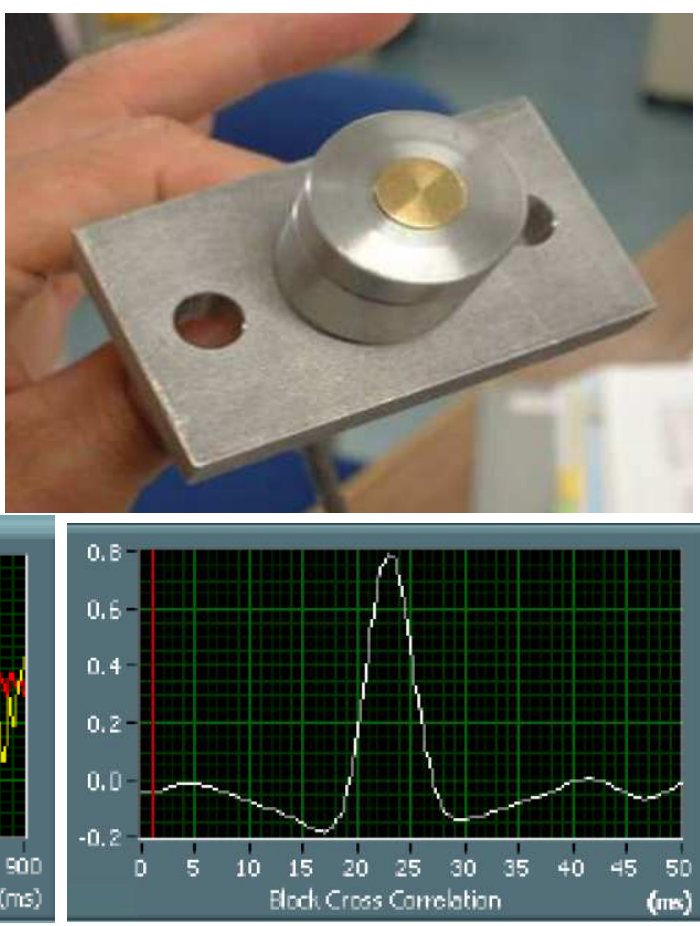

Figure 1: Installation of cross-correlation flow meter on feed-water line of nuclear power plant (top left). High temperature ultrasonic transducer (top right). Two signals representing perturbation of upstream (A) and downstream (B) ultrasonic waves by turbulence (bottom left). Cross-correlation function of the upstream and downstream signals (bottom right). (Courtesy of Advanced Measurement \& Analysis Group Inc.) 


\section{General Form of the Turbulent Field}

Considering turbulent flow in the Cartesian coordinate system $(x, y, z)$ within finite time interval $T$, a turbulent velocity component $v$, or distribution of any other turbulence parameter, can be presented as a Fourier series in the following form:

$$
v(x, y, z, t)=\sum_{j=1}^{M} A_{j}(x, y, z) \cos \left[\omega_{j} t+\theta_{j}(x, y, z)\right],
$$

where $\omega_{j}=2 \pi f_{j}, f_{j}=j / T$, subscript $j$ is the harmonic number, $A_{j}$ and $\theta_{j}$ are harmonic amplitude and harmonic phase respectively, and $t$ is time. Coordinates $x, y, z$ are defined in a flow domain with characteristic size $D$.

It is assumed that time interval $T$ is sufficiently large to allow for accurate representation of the turbulence by Fourier series (1), and the number of harmonics $(M)$ is high enough to include the desired highest turbulence frequencies.

Functions $A_{j}(x, y, z)$ are defined by the turbulence power spectrum, which is a statistical characteristic of turbulence, and typically has spatial scale of the order of the size of the flow domain $D$. The spatial structure of an instantaneous distribution of turbulence parameter $v(x, y, z)$ at a given time is more complicated. It is characterized by a range of scales, from large scale of order of $D$, to smallest scale much smaller than $D$, such as the Kolmogorov's scale [22]. In equation (1) this structure is defined by spatial distributions of phase $\theta_{j}(x, y, z)$.

Methods of measurement of the turbulence spectrum are well developed, and usually sufficient information about functions $A_{j}(x, y, z)$ is available. Further discussion is mainly concerned with the other parameters in (1).

For simplicity, it is assumed further in the paper that time-average velocity has only one non-zero velocity component, in the $z$ direction, and its scale $U$ is significantly higher than the turbulence velocity scale $u$. This assumption allows for using certain relations between time and space scales, such as the Taylor Approximation [15].

\subsection{Time $T$ and the total number of harmonics $M$}

The selection of parameters $T$ and $M$ from (1) depends on the considered problem. However, some general relations between turbulence characteristics and the parameters from (1) can be obtained. 
Representation of turbulence in (1) is based on the assumption that random dependence of a turbulent parameter on time can be approximated by periodical function with period $T$. Such an approximation is valid if $T$ is significantly higher than the time scale of the turbulence, defined by the size of the flow domain $D$ and velocity scale $U$.

$$
T>>\frac{D}{U}
$$

It is convenient to introduce length scale $L$, associated with the time $T$, and parameter $N$ as a ratio of the scale $L$ to $D$, as follows:

$$
L=U T, \frac{L}{D}=N
$$

From (2) and (3), it follows that $N>>1$.

For a given harmonic $j$, a spatial longitudinal scale of turbulence, $\lambda_{j z}$ can be defined by the harmonic frequency and velocity, using the Taylor Approximation [15].

$$
\lambda_{j z}=\frac{U}{f_{j}}
$$

From (1) and (3), it follows that

$$
\lambda_{j z}=\frac{L}{j}
$$

Spatial tangential scale $\lambda_{j \tau}$, in the direction perpendicular to the main flow, is defined by the variation of $\cos \left[\theta_{j}(x, y, z)\right]$. If the total variation of $\theta_{j}(x, y, z)$ on a distance $D$ in tangential direction, $\Delta \theta_{j \tau}$, is of order $2 \pi n$,

$$
\Delta \theta_{j \tau} \sim 2 \pi n(j)
$$

then the spatial scales of the turbulence structure are estimated as:

$$
\lambda_{j \tau} \sim \frac{D}{n(j)}
$$

From (5) and (7) the ratio of the longitudinal and tangential scales is:

$$
\frac{\lambda_{j z}}{\lambda_{j \tau}} \sim N \frac{n(j)}{j}
$$


Therefore, the desired relation between longitudinal and tangential scales can be achieved by selecting an appropriate function $n(j)$ when designing $\theta_{j}(x, y, z)$.

From (8) it also follows that for a given ratio between the longitudinal and tangential scales, the increment of $n$ by one unit corresponds to the increment of $j$ by $N$ units.

$$
\Delta j \sim N \Delta n
$$

Estimation (9) shows that small variation in $n$, and correspondingly small variation in spatial scales, corresponds to a significant number of terms in (1). This result is applicable to both the longitudinal and tangential scales if $\lambda_{j}$ is expressed as a fraction of $D$

$$
\lambda_{j} \sim \frac{D}{n(j)}
$$

From (10), one can conclude that parameter $N$ characterizes the ability of series (1) to approximate the desirable spatial turbulence structures which provide a valid statistical representation of the real turbulent field. This is further discussed in the next section in relation to the two-point correlation.

The total number of terms $M$ in (1) depends on what is the smallest spatial scale $\lambda_{M}$, or what is the highest turbulence frequency $f_{M}$, that is required to be present in the modeling. If $\lambda_{M}$ is presented as a fraction of $D$ as $\lambda_{M}=D / m$, the maximum number of terms in (1) is

$$
M=N m
$$

\subsection{Two-Point Correlation and phase function $\theta_{j}(x, y, z)$}

An important statistical characteristic of a turbulent flow is a correlation between turbulence parameters in two points in space $\vec{\rho}_{0}=\left(x_{0}, y_{0}, z_{0}\right)$ and $\vec{\rho}_{1}=\vec{\rho}_{0}+\Delta \vec{\rho}$, where $\Delta \vec{\rho}=(\Delta x, \Delta y, \Delta z)$.

The two-point correlation $R\left(\vec{\rho}_{0}, \Delta \vec{\rho}, \tau\right)$ is defined as follows:

$$
R\left(\vec{\rho}_{0}, \Delta \vec{\rho}, \tau\right)=\int_{0}^{T} v\left(\vec{\rho}_{0}, t\right) v\left(\vec{\rho}_{0}+\Delta \vec{\rho}, t+\tau\right) d t
$$

If a turbulent field is described by (1), the two-point correlation has the following form:

$$
R\left(\vec{\rho}_{0}, \Delta \vec{\rho}, \tau\right)=\frac{1}{2} \sum_{j=1}^{m} A_{j}^{2} \cos \left[\omega_{j} \tau+\theta_{j}\left(\vec{\rho}_{0}+\Delta \vec{\rho}\right)-\theta_{j}\left(\vec{\rho}_{0}\right)\right]
$$


From (12), the maximum contribution to the magnitude of $R\left(\vec{\rho}_{0}, \Delta \vec{\rho}, \tau\right)$ from each harmonic, corresponds to the following relation between $\tau$ and $\Delta \vec{\rho}$ :

$$
\omega_{j} \tau+\Delta \theta_{j}\left(\vec{\rho}_{0}, \Delta \vec{\rho}\right)=0
$$

Relations (13) can be used to obtain qualitative properties of the spatial behavior of phases $\theta_{j}(\vec{\rho})$ for a typical turbulent flow.

In the two following sections, the spatial behavior of $\theta_{j}(\vec{\rho})$ is considered along the main flow direction, and in the direction perpendicular to the main flow.

\subsubsection{Two Point Correlation in the Longitudinal Direction}

In order to describe the spatial dependence of the phase of the $j$-th harmonic, the phase change $\theta_{j}(\vec{\rho})$ along the flow direction $z$ with scale $\lambda_{j z}$, has to be estimated as follows:

$$
\Delta \theta_{j}\left(z_{0}, \Delta z\right) \sim \Delta z \frac{2 \pi}{\lambda_{j z}}
$$

From (14), (13) and (4), it follows that each harmonic has a maximum contribution to the two-point correlation at the same magnitude of $\tau$ for all harmonics:

$$
\tau \sim \frac{\Delta z}{U}
$$

If (14) is satisfied not approximately, but exactly, then (15) is also satisfied exactly. In this case, the two-point correlation has maximum at time delay $\tau$, defined by (15), and the magnitude of this maximum does not decay with increasing distance $\Delta z$. Such flow corresponds to a frozen turbulence approximation, in which spatial turbulence structures are moving along the main flow direction with constant velocity $U$.

In a realistic description of turbulence, as follows from Kolmogorov's estimations [22], the time of life of a turbulent structure is shorter for smaller scales. This property of turbulent flow results in a decay of the magnitude of the maximum of the two-point correlation, as distance between the points increases.

The above fundamental property of turbulence, combined with equation (14), introduces an apparent paradox, which can be illustrated using the example of isotropic uniform turbulence. In such a model of turbulence, the 
two-point correlation function depends only on distance between two points, and does not depend on the position of these two points in space. This requirement can be satisfied only if dependence of phase $\theta_{j}$ on $z$ is linear. Note that any non-liner relation will result in dependence of $\Delta \theta_{j}$ on position in space. It means that equation (14) has to be satisfied not as a qualitative relation but exactly. That leads to the only possible turbulent field, namely frozen turbulence with a two-point correlation function that does not decay. A resolution to this apparent conflict is given as follows.

Relation (9) shows that if parameter $N$ is a statistically significant number, then equations (1) and (12) have a significant number of terms (of order $N)$, associated with approximately the same magnitude of the turbulence scale, $\lambda_{j} \sim D / n(j)$. Therefore, if phase $\theta_{j}(z)$ depends on a random factor $r_{j}$ for each harmonic, equation (14) does not result in the same magnitude of $\tau$ for each harmonic. At the same time, the turbulence structures described by $N$ consecutive terms may have spatial scale $\lambda_{j} \sim D / n(j)$, which satisfy (14) statistically. Therefore, a different rate of decay of the two-point correlation for different scales can be achieved by introducing a random component $\theta_{r j}(z)$ in the phase function $\theta_{j}(z)$, such that the random effect is increasing on a shorter distance for smaller scales (higher wave number). For example, it can be achieved if the random component of $\theta_{j}(z)$ is described using the random factor $r_{j}$ as follows:

$$
\Delta \theta_{r j}\left(z_{0}, \Delta z\right) \sim r_{j} \Delta z \frac{2 \pi}{\lambda_{j z}}
$$

Relation (16) is presented to qualitatively illustrate the effect of a random factor on the behavior of the two-point correlation function. The specific dependence of $\theta_{j}(z)$ on $r_{j}$ can be selected to obtained the desirable shape of the two-point correlation, depending on the considered problem.

\subsubsection{Tangential Two-Point Correlation}

Phase change along the direction of the vector $\Delta \vec{\rho}$ can be estimated as follows:

$$
\Delta \theta_{j}\left(\vec{\rho}_{0}, \Delta \vec{\rho}\right) \sim\|\Delta \vec{\rho}\| \frac{2 \pi}{\lambda_{j \tau}}
$$

Some properties of functions $\theta_{j}\left(\vec{\rho}_{0}, \Delta \vec{\rho}\right)$ can be obtained by considering isotropic uniform turbulence, as was done in the previous section. For this 
model of turbulence, the two-point correlation depends only on the distance between points and does not depend on the position and orientation of the points in space. To satisfy this condition for each term in (12), the difference $\Delta \theta_{j}\left(\vec{\rho}_{0}, \Delta \vec{\rho}\right)$ between two points separated by distance $\|\Delta \vec{\rho}\|$ has to depend only on $\|\Delta \vec{\rho}\|$ regardless of orientation of vector $\Delta \vec{\rho}$. However, such behavior cannot be achieved in any differentiable function $\theta(x, y, z)$, because the phase change is equal to zero, $\Delta \theta(\Delta x, \Delta y, \Delta z)=0$, along the line perpendicular to the gradient of $\theta(x, y, z)$. A resolution to this apparent paradox is the same as was discussed in Section 2.2.1. Independence of the two-point correlation from $\vec{\rho}_{0}$ and from orientation of $\Delta \vec{\rho}$ can be achieved statistically over a significant number of terms (of order $N$ ) in (12) by introducing a random factor $r_{j}$, as was qualitatively illustrated in (16).

The maximum of the correlation function for a given $\Delta \vec{\rho}$ in the direction perpendicular to the main flow typically corresponds to $\tau=0$, and the magnitude of the value at $\tau=0$ decays as the distance between two points, $\Delta \rho$, increases. From (13) condition $\tau=0$ results in the following condition for the phase:

$$
\Delta \theta_{j}\left(\vec{\rho}_{0}, \Delta \vec{\rho}\right)=0
$$

This condition must also be satisfied statistically over a large number of terms in (12), which correspond to approximately the same turbulence scale.

\section{Specific Form of the Phase Equation and Simulations of Turbu- lent Velocity Fields}

The phase function for each harmonic is assigned as follows:

$$
\begin{aligned}
& \theta_{j}(x, y)=r_{0 j}+n(j) \pi r_{j} \sqrt{\left(x-r_{x j}\right)^{2}+\left(y-r_{y j}\right)^{2}+\epsilon} \\
& \pi \leq\left|r_{0 j}\right| \leq-\pi ; \quad 0.25 \leq\left|r_{j}\right| \leq 1 ; \quad \epsilon<0 ;
\end{aligned}
$$

Parameter $n(j)$ defines the tangential spatial turbulence scale associated with harmonics $j$, as was introduced in (7). $r_{0 j}$ and $r_{j}$ are random factors. The factor $r_{0 j}$ is defined by a uniform distribution between $-\pi$ and $\pi$. The factor $r_{j}$ is defined by a uniform probability distribution between 1 and -1 , but not including interval between 0.25 and -0.25 , to avoid occurrences of very large tangential spatial scales.

Coordinates $(x, y)$ are normalized to a pipe diameter and have magnitude between -0.5 to 0.5 with pipe center at $(x=0, y=0)$. Expression (19) generates the spatial distribution of each harmonic in the shape of 
an oscillating function with central symmetry at a randomly selected point $\left(x=r_{x j}, y=r_{y j}\right)$.

In the case that the randomly selected point $\left(x=r_{x j}, y=r_{y j}\right)$ is within the flow domain, a singularity exists at this location, as the phase for harmonic $j$ will not be a smooth function over the spacial domain of the flow. In order to remove this singularity, the term $\epsilon$ is included in (19). If point $\left(x=r_{x j}, y=r_{y j}\right)$ is assigned outside of the flow domain, restrictions must exist that define how far from the flow domain, and how close to the flow domain, the point can be assigned.

An example of level lines of five phase functions for $j=1 \ldots 5$ is shown in Fig. 2.

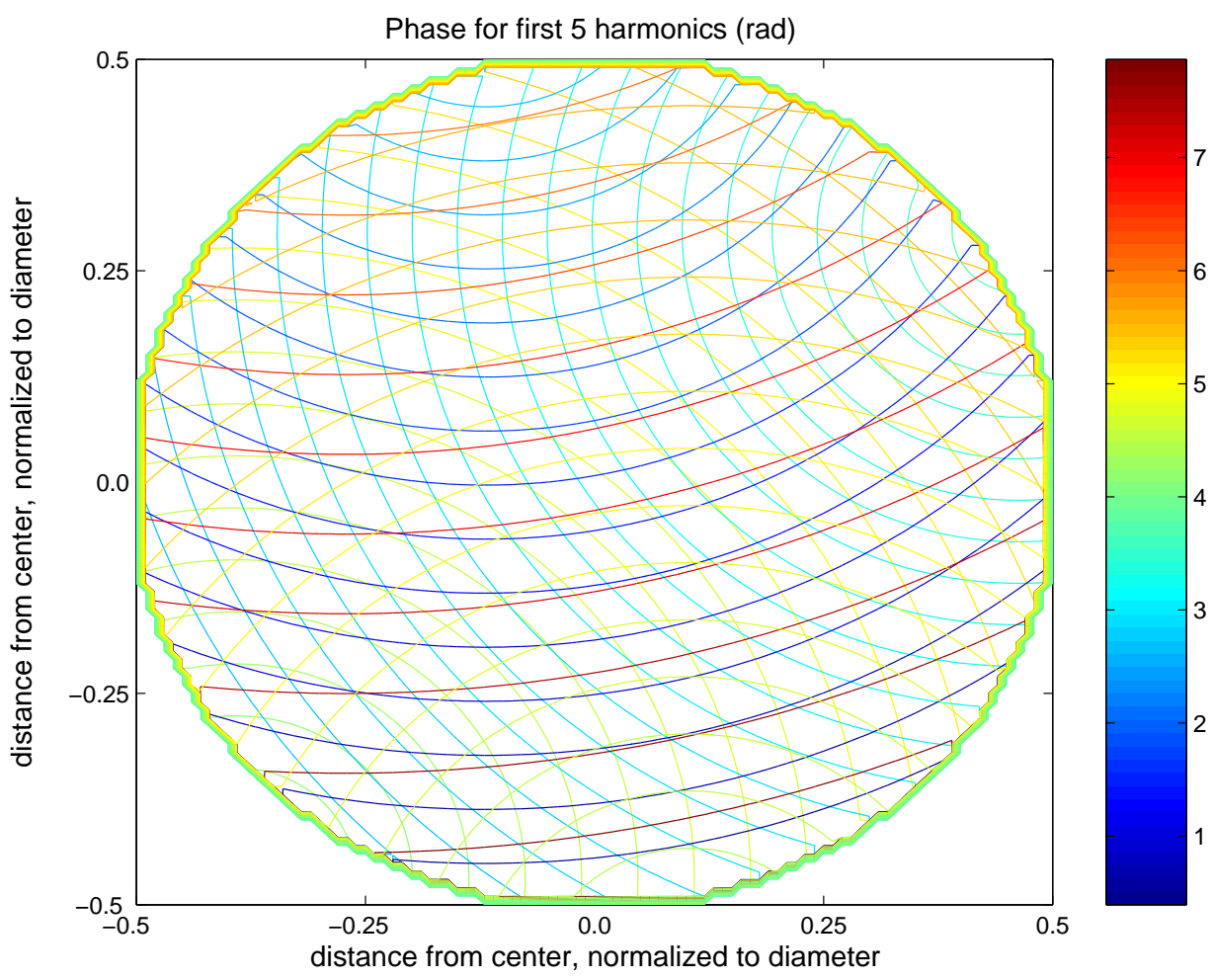

Figure 2: Level lines of phase functions for $j$ equal from 1 to 5 . 
In further examples, function $n(j)$ is selected as follows:

$$
n(j)=k \sqrt{\left(\frac{j}{N}+2.5\right)}
$$

Parameter $k$ is a constant introduced to control the relation between the longitudinal and tangential turbulence scales, as was estimated in (8). Further examples are provided for a pipe flow with the following flow conditions and modeling parameters:

$\begin{array}{ll}\text { Pipe diameter } & D=304.8 \mathrm{~mm} \\ \text { Cross-section average flow velocity } & U=5 \mathrm{~m} / \mathrm{s} \\ \text { Time duration } & T=4 \mathrm{~s} \\ \text { Minimum longitudinal turbulence scale } & \lambda_{m}=D / 32=9.5 \mathrm{~mm} \\ \text { Length scale } & L=U T=24 \mathrm{~m} \\ \text { Parameter } & N=L / D \sim 78.7 \\ \text { Number of harmonics } & M \approx L / \lambda_{m}=2520 \\ \text { Maximum frequency } & f_{M} \approx U / \lambda_{M} \approx 630 \mathrm{~Hz}\end{array}$

The amplitudes $A_{j}(x, y)$ are obtained from measurement of the corresponding pipe flow. Specifically, cross-correlation flow measurement returns the function $\phi(t)$, which is an integral of instantaneous turbulent velocity component $V_{t}(x, y, t)$, as follows:

$$
\phi(t) \sim \int_{0}^{D} V_{t}(x=0, y, t) d y
$$

The spectrum of function $\phi(t)$ is used to obtain the amplitudes $A_{j}(x, y)$ for flow simulation. A more detail discussion of function $\phi(t)$ is presented in Section 4.

Measurement of the spectrum, used in the modeling, was conducted over a long time interval, much longer than modeling interval $T=4 s$. Therefore, amplitudes $A_{j}$ in the modeling did not include any random factor. Consequently, random variations between turbulent fields obtained in different modeling runs were expected to be smaller than in the real flow. For simplicity, it was also assumed that amplitudes $A_{j}$ are not changing along the pipe cross section.

Each simulation run corresponds to 4 seconds of turbulent flow over a given pipe cross-section. Similarly to a real turbulent flow, each run results 
in a unique turbulent field defined by a unique set of phase functions, due to the presence of random parameters. How well statistical characteristics of the turbulent field are represented in a single run, depends on the number $N$. As follows from (9), and as was discussed in Sections 2.2.1 and 2.2.2, the higher the number $N$, the higher the number of harmonics are available to describe spatial turbulence structure in a certain range of the turbulence spectrum, or in a certain range of spatial scales. In the present example, the range of longitudinal scales from $\lambda_{\tau} \sim 1 D$ to $\lambda_{\tau} \sim 0.9 D$ corresponds to the harmonic number range $j$ from 1 to approximately 50 . Thus, there are approximately 50 terms available in equation (1) to describe this portion of the turbulence structures. To illustrate this, Fig. 3 presents an example of level-lines of 50 phase functions from $j=1$ to $j=50$, superimposed on each other.

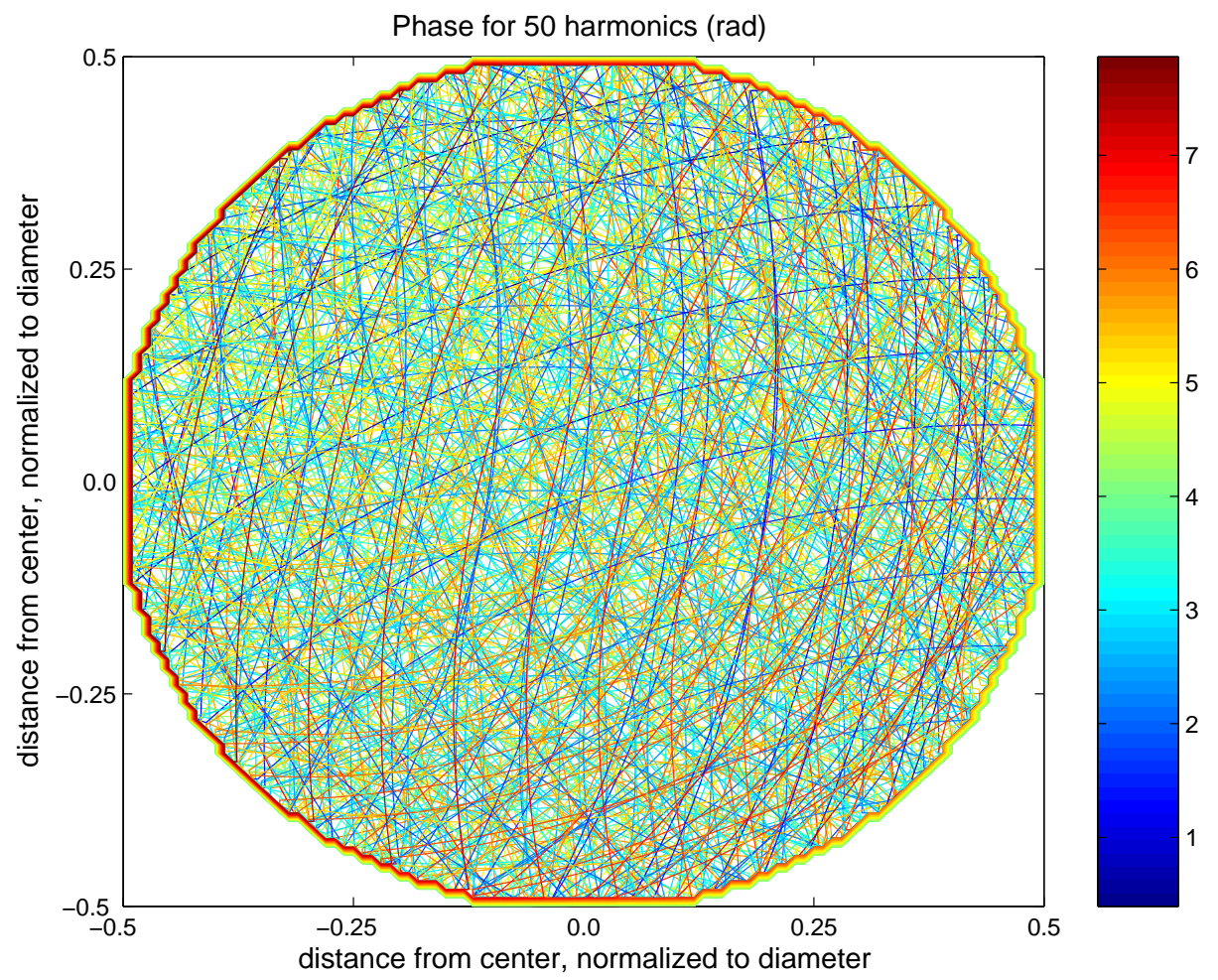

Figure 3: Level-lines of 50 phase functions, corresponding to harmonics numbers from $j=1$ to $j=50$. 
Figure 4 presents simulated phase at the center of the pipe, for each harmonic from $j=1$ to $j=2520$, for three different modeling runs, each with different values of constant $k$ from equation (20), $k=1$ (Blue), $k=2$ (Red) and $k=4$ (Black).

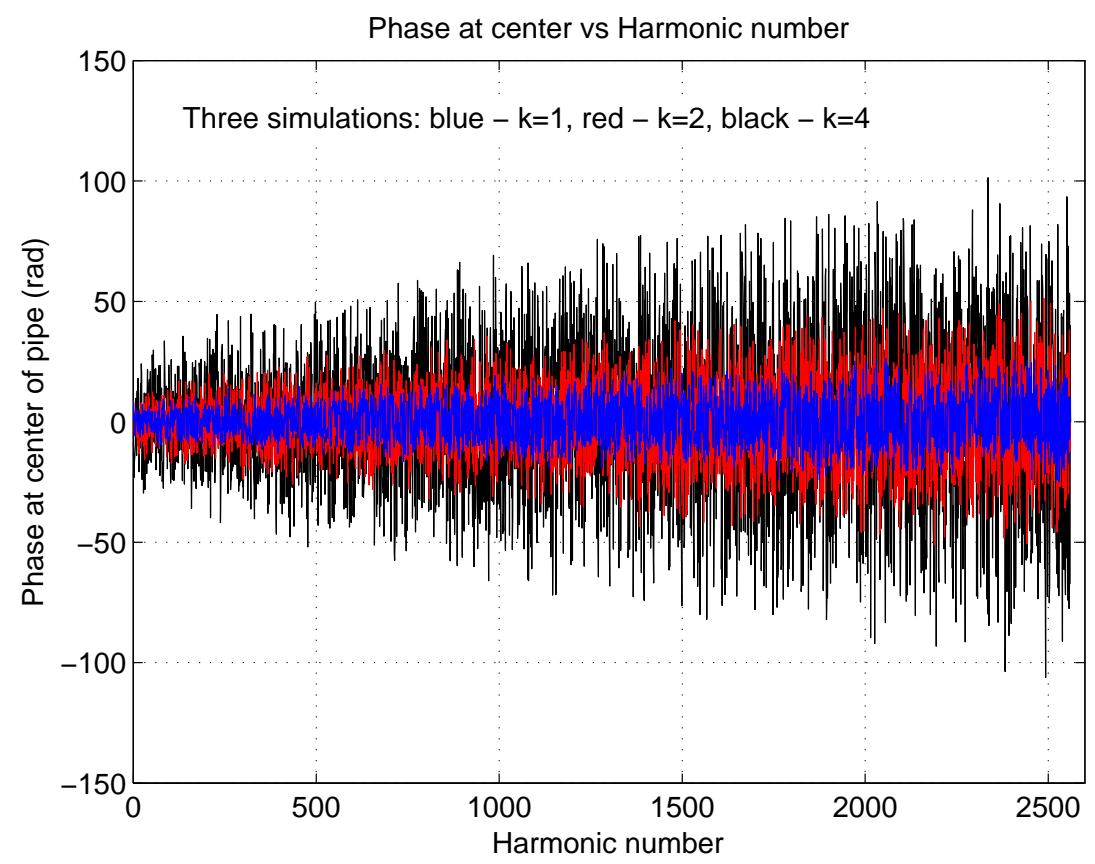

Figure 4: Phase at the center of the pipe, for each harmonic, for three different modeling runs with $k=1$ (Blue), $k=2$ (Red) and $k=4$ (Black).

Fig. 4 shows that the range of the phase values, at the center of the pipe, increases with increase of harmonics number. The rate of the increase can be controlled by parameter $k$. However, variation of the phase change between two consecutive harmonics can be significant. Similar results can be shown for locations other than the center of the pipe. The lower frequency structures will have higher spacial correlation, in the tangential direction, than higher frequency structures. The rate of decrease of spacial correlation increases as $k$ increases. As a result of these properties, the selection of a $k$ value, allows one to control the tangential spacial scales of simulated turbulent structures.

Level-lines of the velocity field in the $(x, t)$ plane are shown in Fig. 5 for $k=2$ (top plot) and $k=4$ (bottom plot). Level-lines in the $(x, y)$ plane at $t=$ constant for the same simulations are shown in Fig. 6, for 
the same $k$ values respectively. Fig. 5 and Fig. 6 illustrate how the ratio between longitudinal and tangential scales, and the tangential scale itself, can be controlled by parameter $k$.
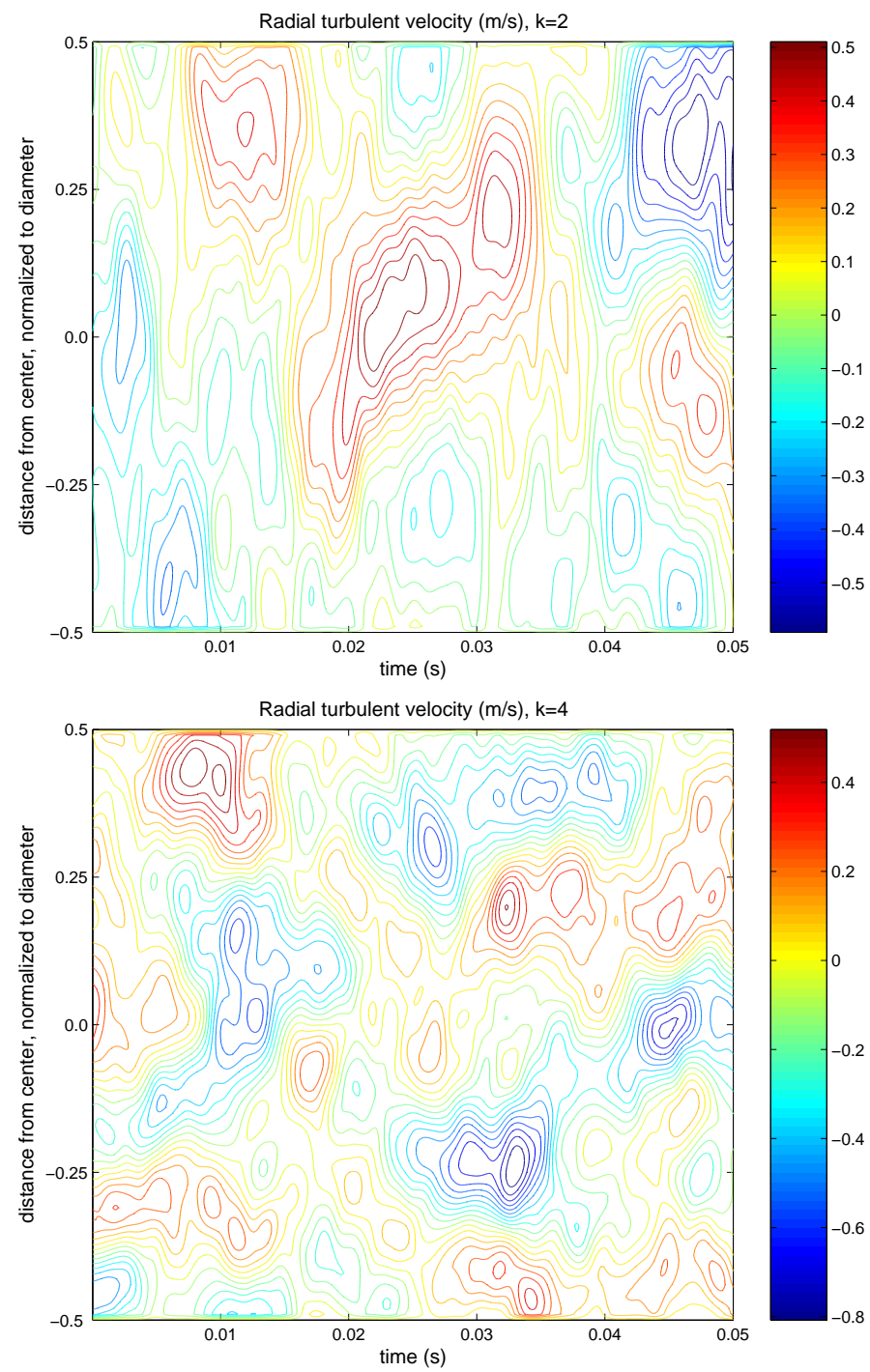

Figure 5: Velocity level-lines in $(x, t)$ plane for $k=2$ (top) and $k=4$ (bottom) 

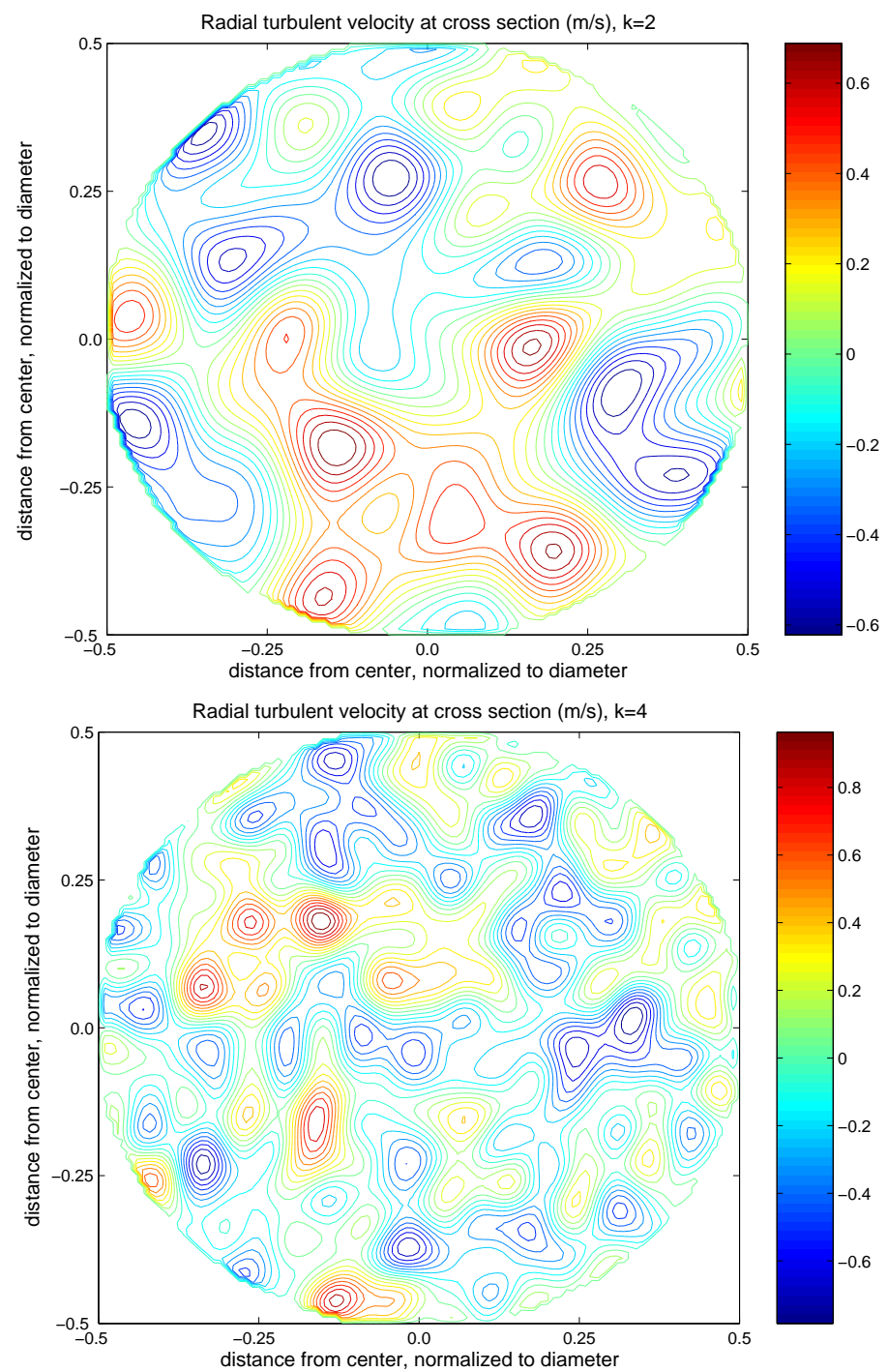

Figure 6: Velocity level-lines in $(x, y)$ plane at $t=$ const, for $k=2$ (top) and $k=4$ (bottom).

An example of the two-point correlation function described by (12), is shown in Fig. 7. In this example $\vec{\rho}_{0}=(0,0,0)^{\prime}$ and $\Delta \vec{\rho}=(0, \Delta y, 0)^{\prime}$. Fig. 7 shows results for simulation runs with $k=2$ (top plot) and $k=4$ (bottom plot). Both the top and bottom plots in Fig. 7 shows a family of ten curves corresponding to different distances $\Delta y$, where $y$-values are 
normalized to pipe diameter, and $y=0$ corresponds to the center of the pipe. The top curve in both plots corresponds to $\Delta y=0.01$. The remaining nine curves correspond to $\Delta y=0.05,0.1,0.15,0.2,0.25,0.3,0.35,0.4$, and 0.45 , respectively. For greater $\Delta y$, the corresponding correlation curve has a lower peak, and for large enough $\Delta y$ there is no peak at all. The rate at which the peak drops with increasing $\Delta y$, will be greater for larger values of $k$.

The dependence of the value of the two-point correlation at $\tau=0$, on distance $\Delta \vec{\rho}=(0, \Delta y, 0)^{\prime}$ along the pipe radius is shown in Fig. 8, for $k=2$ and $k=4$. Behavior of the two-point correlation is consistent with illustrations in Fig. 5, Fig. 6, and Fig. 7. The value of the correlation at $\tau=0$ reduces over shorter distances, for higher values of parameter $k$, which correspond to a flow with smaller turbulence scales.

It should be noted, that the simulations presented in Fig. 7 and Fig. 8 correspond to the same user specified simulation parameters, but are not the exact same simulation. Therefore, since the simulation has random components, Fig. 7 and Fig. 8 present results with the same qualitative characteristics, and similar quantitative characteristics, but not identical quantitative characteristics.

Fig. 9 shows experimental data presented by Taylor [23] on a $73 \mathrm{~mm}$ diameter pipe at a long distance from the pipe inlet. Experimental data can be reproduced via mathematical simulation by adjusting parameter $k$. Good agreement between modeling and experimental results shown in Fig. 9 is obtained with simulation using $k=5$.

In general, results presented in Figs. 5 through 9 show that ratio between the longitudinal and tangential turbulence scales, and behavior of the twopoint correlation, can be effectively controlled by parameter $\mathrm{k}$. 

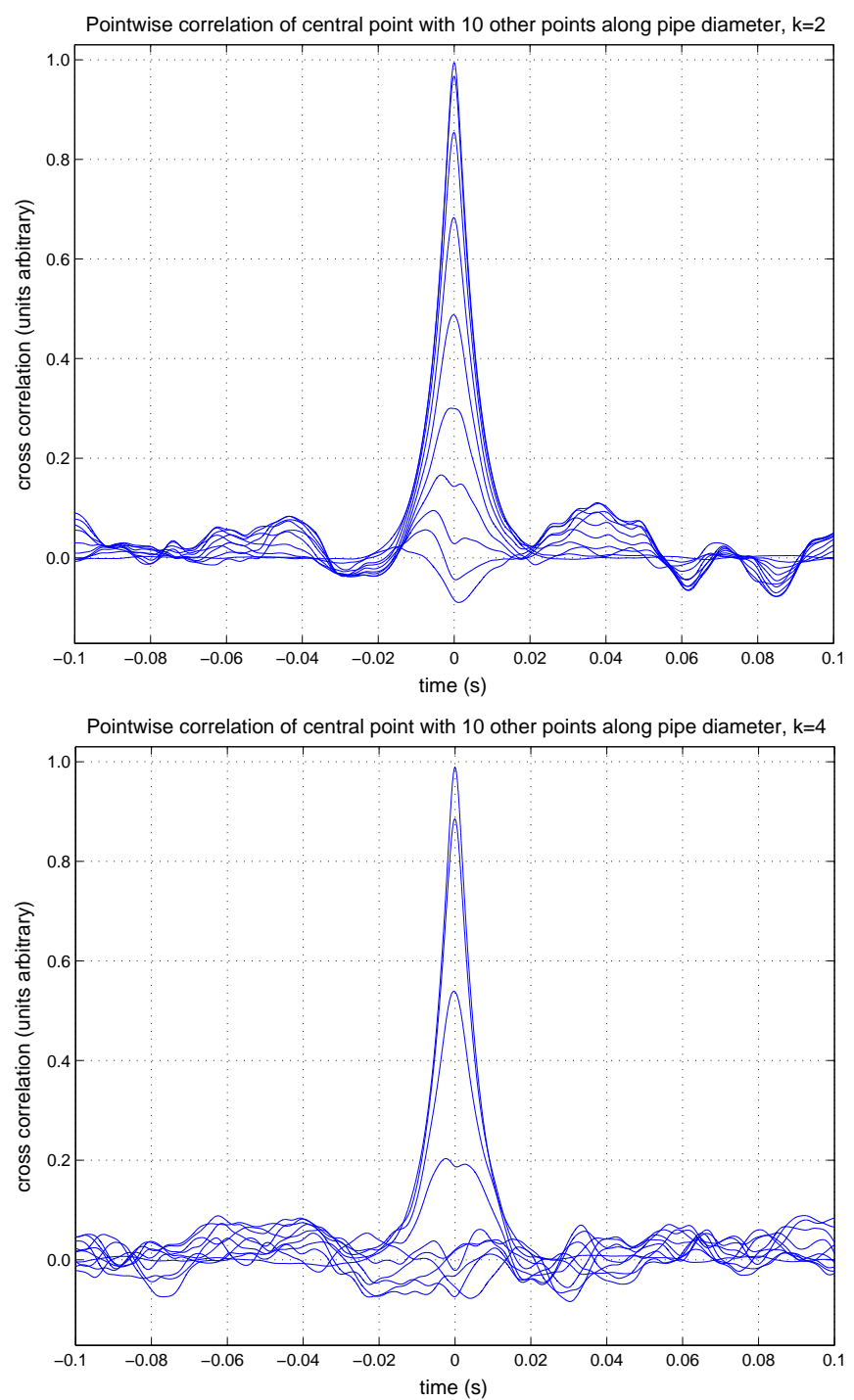

Figure 7: Dependence of two-point correlation (12) on $\tau, k$, and $\Delta y$. One point is located at the center of the pipe; the other points are located along the $y$-axis with $\Delta y=0.01,0.05,0.1,0.15,0.2,0.25,0.3,0.35,0.4$, and $0.45 . k=2$ (top), $k=4$ (bottom). 


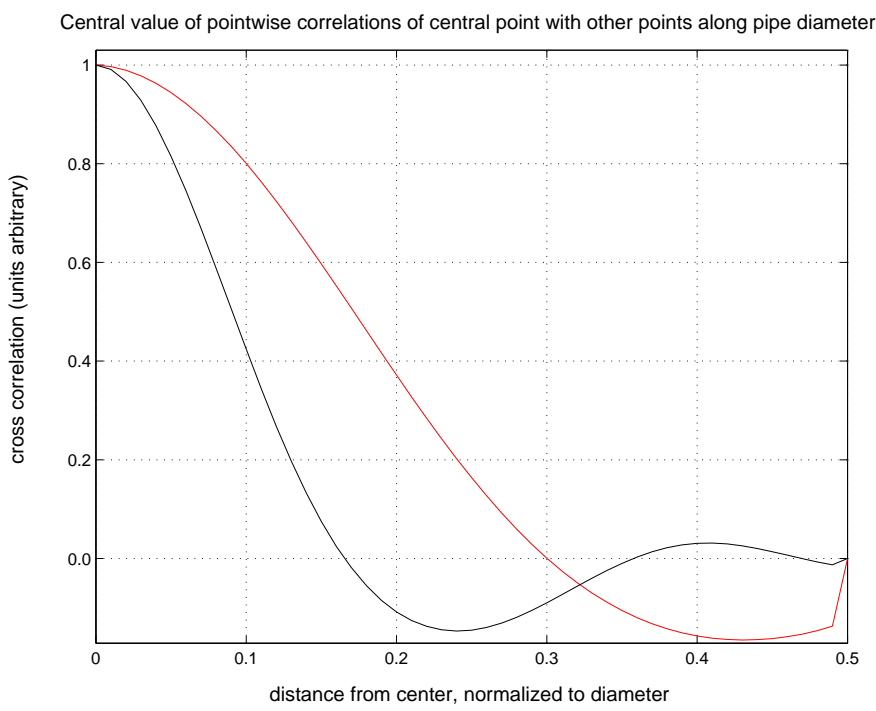

Figure 8: Distribution of two-point correlation function (12) at $\tau=0$ along $y$ axis when one point is fixed at the center of the pipe. The two curves represent two different simulation runs, with $k=2$ (red) and $k=4$ (black).

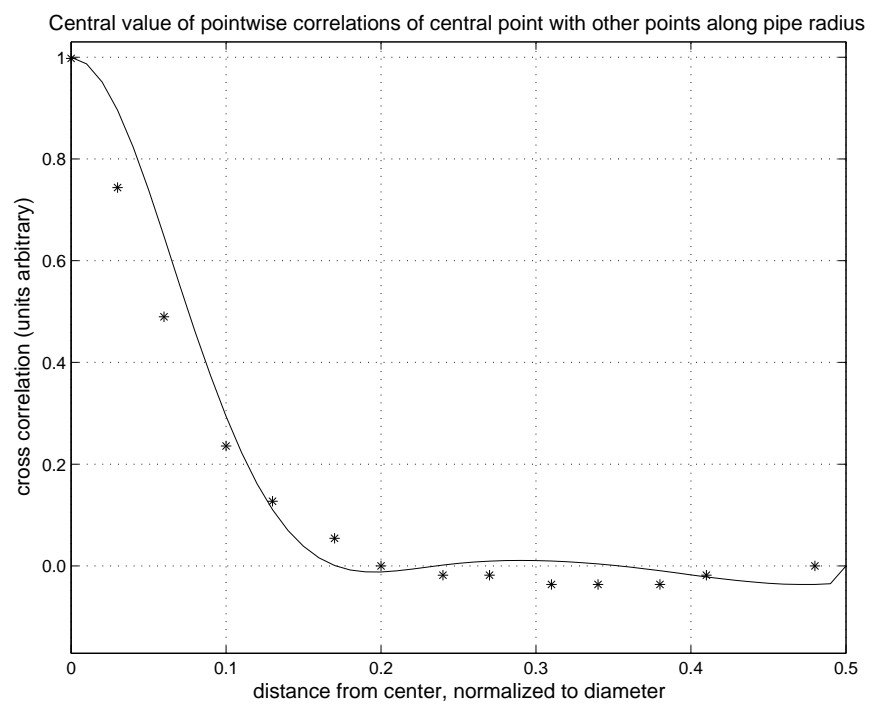

Figure 9: Comparison of simulated and experimental two-point correlation functions at $\tau=0$ along $y$ axis when one point is fixed at the center of the pipe. Solid line - simulation at $k=5$, Stars - experimental data from Taylor [23]. 


\section{Application of the Modeling Method to Mathematical Simula- tion of Cross-Correlation Flow Measurement}

The synthetic turbulence model described above was used for mathematical simulation of cross-correlation flow measurement. Results of the simulations were compared with experimental data. Principles of the crosscorrelation flow measurement method, along with the results of mathematical simulation and experimental data are discussed in the following sections.

\subsection{Cross-Correlation Flow Measurement Technology}

The ultrasonic cross-correlation flow rate measurement method has been known for a long time [16], but it became more popular in recent decades due to advancement of modern computers [17, 18, 19, 20, 21].

In the most simple realization of the ultrasonic cross-correlation flow meter, two ultrasonic waves, separated by a known axial distance, are transmitted through the flow along pipe diameter (see Fig. 10). Each wave is modulated by turbulent structures which are naturally present in the flow. Phase delay of the ultrasonic wave is modulated by the turbulence velocity component along direction of propagation of the ultrasonic waves according to the following equation $[19,20,21]$ :

$$
\phi(t)=\frac{f_{U}}{c^{2}} \int_{0}^{D} V_{t}(\zeta, t) d \zeta
$$

In the integral (22) $f_{U}$ is frequency of the ultrasonic wave, which has a magnitude of order $1 \mathrm{MHz}$, significantly higher than turbulent spectrum frequencies, $c$ is the speed of sound, $V_{t}$ is the turbulence velocity component along the direction of wave propagation, and $\zeta$ is the spatial variable of integration along the direction of wave propagation. If signal $\phi(t)$ is obtained at two different cross-sections of the pipe, $z=Z_{1}$ and $z=Z_{2}$, the cross-correlation function of signals $\phi\left(Z_{1}, t\right)$ and $\phi\left(Z_{2}, t\right)$ can be calculated as follows, where $T$ is the time duration of the signals:

$$
R(\tau)=\int_{0}^{T} \phi\left(Z_{1}, t\right) \phi\left(Z_{2}, t+\tau\right) d t
$$

If the distance between the two ultrasonic beams, $l=Z_{2}-Z_{1}$, is short enough, then the signals $\phi\left(Z_{1}, t\right)$ and $\phi\left(Z_{2}, t\right)$ maintain similarity to each 
other. In this case, function $R(\tau)$ has a pronounced maximum at $\tau=\tau^{*}$ (See Fig. 1, bottom right plot). The measured flow velocity $V_{m}$ is defined as:

$$
V_{m}=\frac{l}{\tau^{*}}
$$

The meaning of $V_{m}$ can be better understood from the following considerations. Function $R(\tau)$ can be presented as a double integral of the two-point correlation functions by substituting (22) into (23), and changing the order of integration (see Fig. 10) [20, 21]:

$$
\begin{aligned}
& R(\tau)=\int_{0}^{D} \int_{0}^{D} R_{0}(\zeta, \xi, \tau) d \zeta d \xi \\
& R_{0}(\zeta, \xi, \tau)=\int_{0}^{T} V_{t}\left(Z_{1}, \zeta, t\right) V_{t}\left(Z_{2}, \xi, t+\tau\right) d t
\end{aligned}
$$

Function $R_{0}(\zeta, \xi, \tau)$ is a correlation of turbulent velocity component $V_{t}$ in two points located along direction of propagation of ultrasonic waves at two pipe cross-sections $z=Z_{1} ; y=\zeta$ and $z=Z_{2} ; y=\xi$. From (25) it follows that velocity $V_{m}$, defined by (24), is a weighted average of velocities defined by the positions of the maximums of the two-point correlations $R_{0}(\zeta, \xi, \tau)$. Therefore, under the presence of a time-averaged velocity gradient in a pipe flow, $V_{m}$ is usually not equal to the cross-section average axial flow velocity $U$. In cross-correlation flow measurement, the flow rate is calculated as follows:

$$
Q=C A \frac{l}{\tau *}=C A V_{m}
$$

In equation (26), $Q$ is volumetric flow rate, $A$ is pipe cross-section area, $l$ is spacing between the upstream and downstream ultrasonic beams, $\tau *$ is the position of the maximum of the cross-correlation function, and $V_{m}$ is the measured velocity as it was defined in relation (24). Hydraulic factor $C$ is defined as a ratio of the cross-section average flow velocity $U$ to the measured velocity $V_{m}$.

$$
C=\frac{U}{V_{m}}
$$

Since $V_{m}$ is a measure of the average transport velocity of turbulent structures, the hydraulic factor $\mathrm{C}$ is a measure of the ratio between the crosssection average flow velocity and the average transport velocity of turbulent structures. Determining the value of the hydraulic factor for different flow conditions, and for different design parameters of the flow meter, is the most 


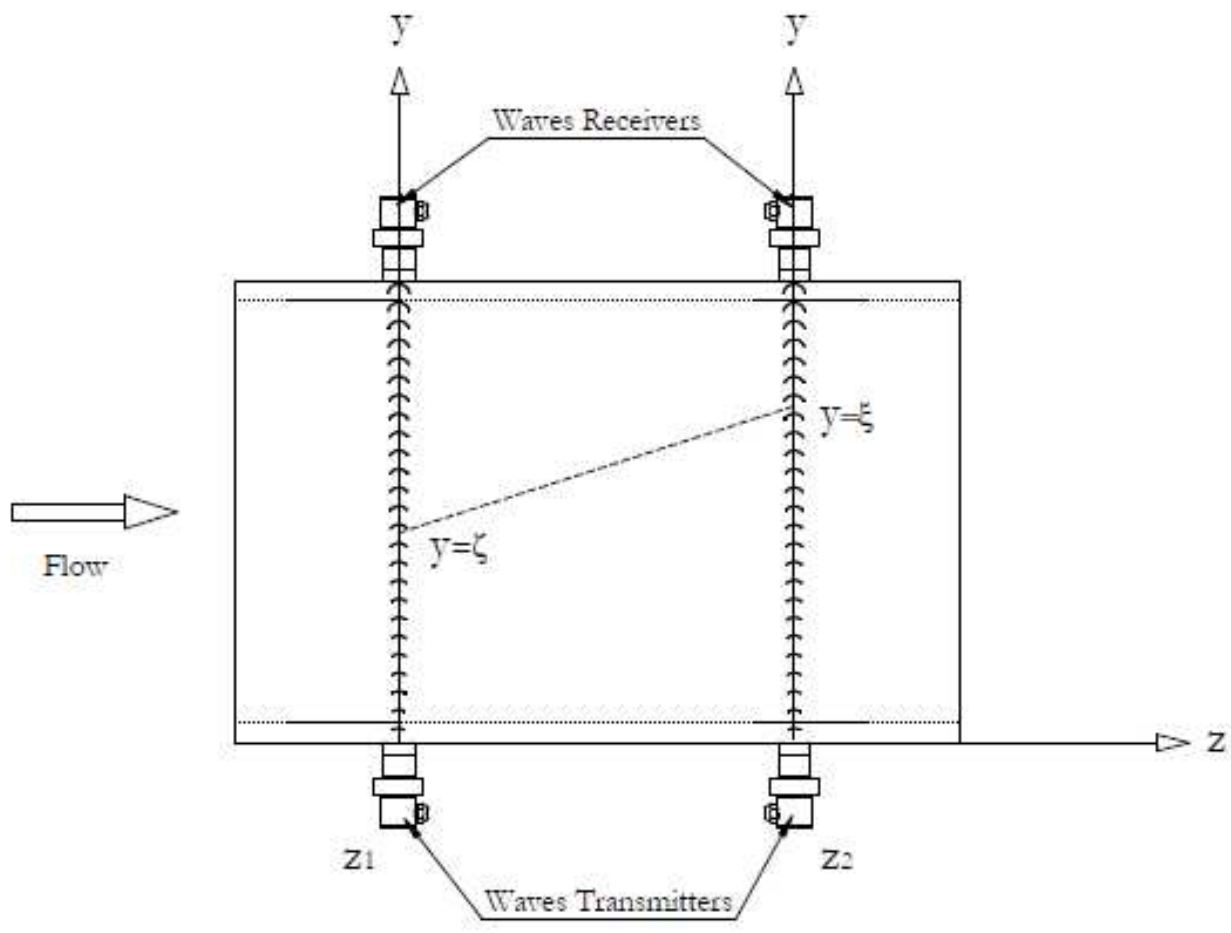

Figure 10: Schematics of cross-correlation flow measurement. Function $R_{0}(\zeta, \xi, \tau)$ is a two-point correlation of velocity $V_{t}$ taken between the points $(x, y)=\left(\zeta, Z_{1}\right)$ and $(x, y)=$ $\left(\xi, Z_{2}\right)$.

important and challenging tasks in cross-correlation flow measurement. The model described in this paper can be used to investigate the sensitivity of the hydraulic factor to different flow conditions, and to predict the hydraulic factor for a specified flow condition. 


\subsection{Mathematical Simulation for Cross-Correlation Flow Measurement}

Mathematical simulation of cross-correlation flow measurement can be used to investigate the effect of different flow conditions and flow meter design parameters, on hydraulic factor $C$.

Another objective of the simulation can be to predict flow meter behavior at a specific flow conditions. Other than the obvious practical interest, such simulation is an important step in evaluation of the mathematical simulation itself, by comparing simulated results with experimental results. For this application, an important requirement is for the simulated flow conditions to be representative of those present at the flow meter location during the experiment. The design of the cross-correlation flow meter used in the experiments described in this section, allows for the recording of signal $\phi(t)$ defined in (22). Statistical characteristics of this signal can be used to determine input parameters for the simulation of the inlet turbulent velocity field, ensuring that the remaining simulation is based on the same flow condition as the experiment.

To compare simulation results with laboratory experiment results, the simulation was conducted at different distances downstream of a 90-degree elbow with two different spacing values between ultrasonic beams: $l=1 D$ and $l=3 D$, where $D$ is the pipe diameter. These spacing values were chosen, because a typical installation of a cross-correlation flow meter places the upstream and downstream ultrasonic beams at a distance ranging from $1 D$ to $3 D$. Analysis of instrument and model performance for spacing values smaller than $1 D$ and greater than $3 D$ is a subject of future work. Results

of the simulation were compared with test data. The simulation consisted of the following steps:

- Numerical simulation of the time-averaged velocity profiles at different distances downstream of the 90-degree elbow, for a given pipe diameter $D$ and cross-section area average flow velocity $U$, using the $k-\epsilon$ model and an ANSYS CFD package.

- Generation of the inlet turbulent velocity field at a given pipe crosssection $z=Z_{1}$ (see Fig. 10) using the method of turbulence field generation described in this paper, and statistical characteristics of the power spectrum of signal $\phi(t)$ from (22) as input for the simulation. 
- Calculation of the turbulence velocity field in cross-section $z=Z_{2}$ (see Fig. 10) using the local Taylor approximation [15, 20, 21].

- Calculation of the measured flow velocity $V_{m}$ using equations (22-24), and calculation of the hydraulic factor $C=U / V_{m}$.

In order to ensure that the simulated turbulence velocity fields were representative of the flow conditions present during laboratory testing, the spectrum of signal $\phi(t)$ from (22) was recorded during the laboratory test. For each flow condition, an ensemble of such power spectra were collected and averaged to remove random noise, revealing the turbulence spectra representative of that particular flow condition. The averaged spectra were used as input for the simulation to calculate amplitudes of the harmonics in (1). It was assumed that the turbulence spectrum is constant across the pipe cross-section area, except for a small area near the pipe wall.

\subsection{Laboratory Test Set-Up}

Comparison of the hydraulic factor values predicted by the simulation and observed in a laboratory test, was conducted using two sets of data: one set obtained in the Utah Water Research Laboratory (UWRL), and the other set obtained in the AMAG flow laboratory in Canada.

In a laboratory test, the volumetric flow rate in a pipe is measured by a reference laboratory instrument, and the cross-section average flow velocity $U$ can be calculated. Installation of a cross-correlation flow meter on the same pipe provides measured velocity $V_{m}$, and consequently the value of the hydraulic factor $C=U / V_{m}$ can be obtained.

In the UWRL facility, water flows through a test section from a natural lake, located approximately 10 meters above the laboratorys altitude, and can be directed into a high capacity weight-tank, as is illustrated in Fig. 11. Time duration of the each test-run was approximately 30 minutes. During this time-interval, weigh-tank measurements were taken every 5 minutes, and average flow rate over the duration of the test-run was calculated. Accuracy of the weigh-tank flow measurement was $0.15 \%$. Transducers of the ultrasonic cross-correlation flow meter were located at different distances downstream of a 90-degree elbow on a 12-inch plastic pipe. Spacing between ultrasonic beams in all UWRL tests was $l=1 D$. 


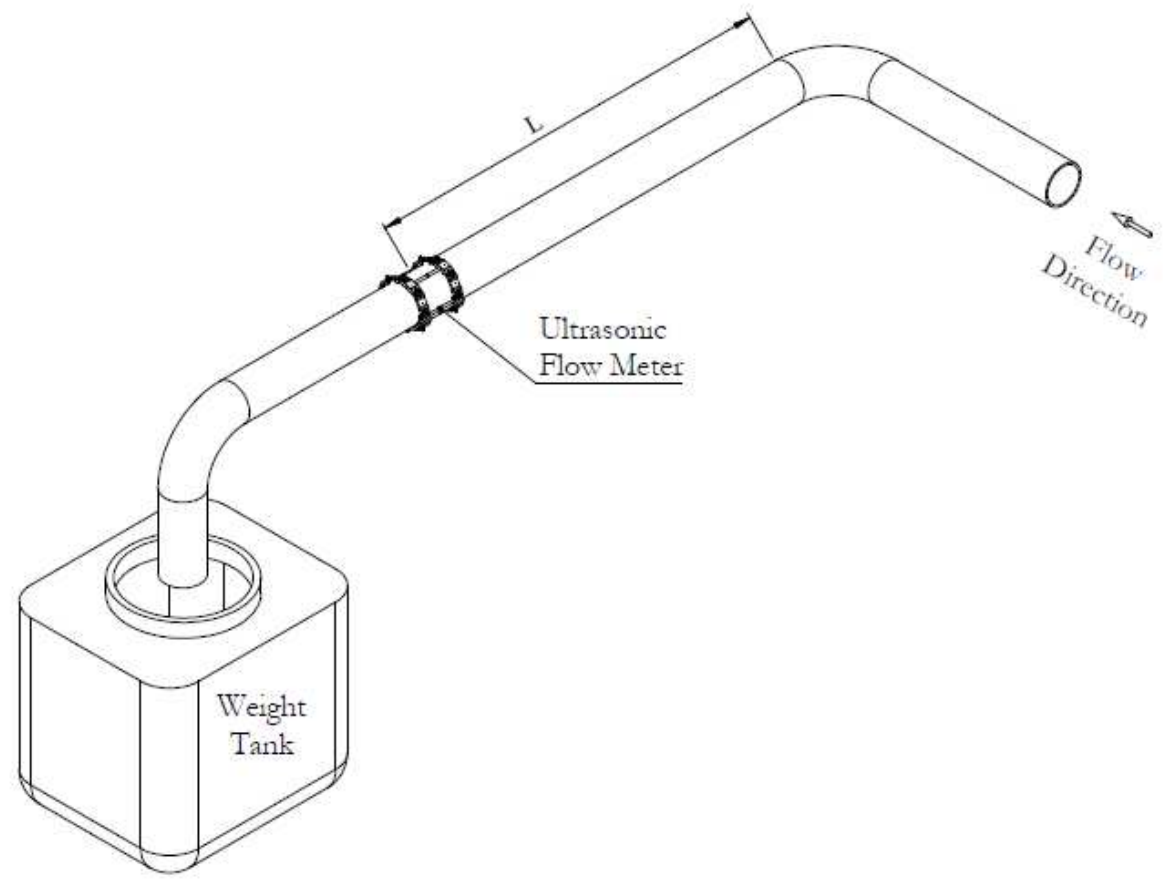

Figure 11: Schematics of the test setup. The test section consists of a 12-inch plastic pipe, with carbon steel 90-degree elbow and flow conditioner.

The second set of data was obtained in the AMAG flow laboratory where flow measurements were conducted with two different spacing values between ultrasonic beams, $l=1 D$ and $l=3 D$, on a 4 -inch plastic pipe, at different locations downstream of the 90-degre elbow. Accuracy of the reference flow measurement instrument was $0.4 \%$. The purpose of this test was to measure the dependence of the hydraulic factor on spacing between ultrasonic beams, and compare this measured spacing dependence to the spacing dependence predicted by the mathematical simulation. 


\subsection{Comparison of the Mathematical Simulation with Laboratory Test Re- sults}

Hydraulic factor $C$, predicted by the mathematical simulation and measured in the UWRL test, is shown in Fig. 12. One can see that the predicted change in $C$ value, over a distance from 20 to 50 pipe diameters downstream from the 90-degree elbow, is slightly higher than the measured change in $C$ value. However, the difference between measured and simulated values of $C$ at each point is small, and within the uncertainty of the measurement, which includes accuracy of the weigh-tank and other uncertainty components associated with the ultrasonic flow meter.

Comparison of the simulated and measured values of $C$ at different distances downstream of a 90-degree elbow with different spacing values between ultrasonic beams, $l=1 D$ and $l=3 D$, is shown in Fig. 13. Experimental data presented in Fig. 13 were obtained at the AMAG flow laboratory.

Results presented in Fig. 13 for spacing $l=1 D$ (blue dots and blue triangles) are consistent with the results obtained in UWRL (Fig. 12). The simulated change of the $C$ value over a distance from 20 to 50 pipe diameters from the elbow is slightly higher than the measured value, and the difference between predicted and measured values of $C$ at each point is within uncertainty of the test which includes the accuracy of the reference flow instrument, and other uncertainty components associated with the ultrasonic flow meter used in the tests. Maximum difference, approximately $1 \%$, is observed on the distance of 50D from the elbow.

Data presented in Fig. 13 on the effect of spacing between ultrasonic beams, $l$, on the hydraulic factor $C$, show that a change of spacing from $l=1 D$ (blue) to $l=3 D$ (red), results in reduction of both the measured (circles) and predicted (triangles) value of $C$. However, the simulation shows a slightly smaller effect than what is shown in the test result. Comparing the most upstream measurement location along the test section to the most downstream measurement location along the test section, the predicted value of $C$ shows a change of approximately $2 \%-3 \%$, and the measured value of $C$ shows a change of approximately $3 \%-4 \%$.

Fig. 13 also shows that the difference between predicted and measured values of $C$ for $l=3 D$ is higher than for $l=1 D$. A possible explanation for this observation, is that the simulated transformation of the turbulence field between the two ultrasonic beams is described by the Taylor approximation $[15,20,21]$, the accuracy of which reduces with increase of spacing $l$. 


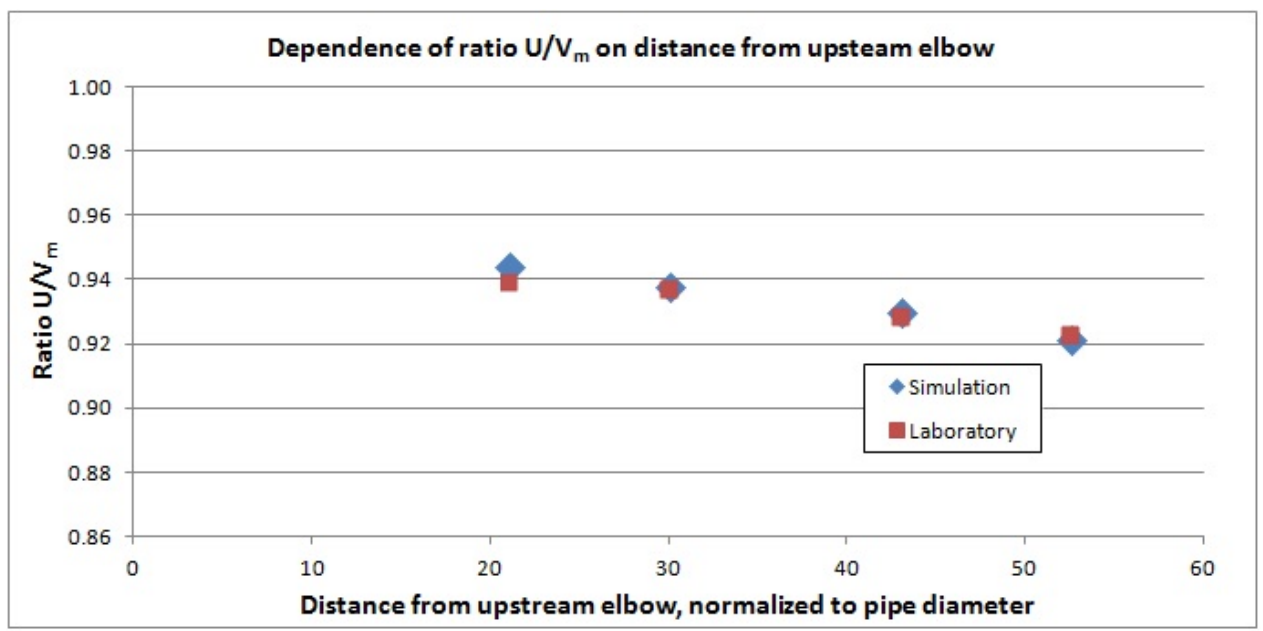

Figure 12: Measured and predicted dependence of ratio $U / V_{m}$ on distance from upstream 90-degree elbow. Diamonds - mathematical modeling, Squares - laboratory test

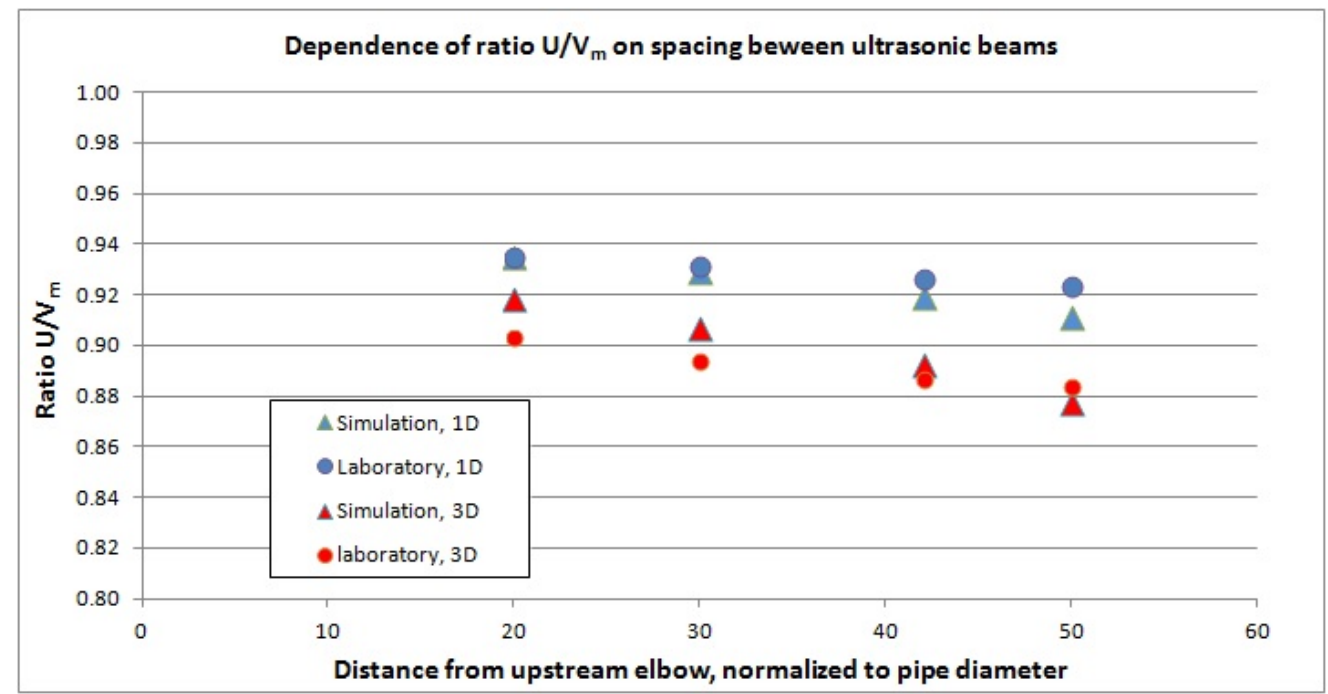

Figure 13: Measured and predicted dependence of ratio $U / V_{m}$ on normalized distance from upstream elbow $L / D$, for two different spacing values between ultrasonic beams. Blue 1D spacing, Red - 3D spacing, Triangles - mathematical model, Circles - laboratory test 


\section{Conclusions}

The new method of generating synthetic turbulence fields, for prediction of ultrasonic cross-correlation flow meter performance under different flow conditions, is presented. The process of selection of the turbulence model parameters for generating desirable spatial characteristics of turbulence, such as distribution of the longitudinal and tangential turbulent scales and distribution of the two-point correlation, is outlined.

The application of the proposed method to turbulent pipe flow, demonstrates good prediction of the two-point correlation and its decay with increase of distance. The simulated behavior of the cross-correlation flow meter demonstrated good prediction of flow meter behavior under different flow conditions and for different distances between the ultrasonic beams of the flow meter. This model allows for real turbulence characteristics to be reproduced by appropriately selecting values of control parameters.

It is expected that the proposed turbulence modeling method will be used as a simulation tool for validation and improvement of cross-correlation flow measurement technology. The method can also be used for evaluation of the effect of turbulence on other flow instrumentation.

\section{Acknowledgments}

The authors acknowledge the support of Advanced Measurement \& Analysis Group Inc. (AMAG) for provision of the ultrasonic cross-correlation flow meter and laboratory facilities. The authors express gratitude to Professor David Novog from McMaster University, Canada, who dedicated significant time for reviewing the project, and provided valuable comments and suggestions. The authors also express gratitude to Professor Stephen Tullis from McMaster University, for important discussions which significantly influenced the direction of this research. Many thanks also go to Professor M. Johnson and his colleagues at Utah State University, USA, for excellent organization of the experiments conducted at the Utah State University Water Research Laboratory in cooperation with AMAG. 


\section{Author Biographies}

Below are brief descriptions of the authors.

Dr. Alexander Gurevich

Dr. A. Gurevich completed his Doctorate in Mechanical Engineering at De Montfort University, specializing in Fluid Dynamics, in 2013, and his Bachelors of Mathematics, Physics Option, at the University of Waterloo in 2008. Since 2009, Dr. A. Gurevich has been engaged in research and development at the Canadian company Advanced Measurement \& Analysis Group Inc., focusing on improvement of ultrasonic cross correlation flow measurement technology, mainly by studying the effect of flow conditions on flow measurement instruments. His current interests are in turbulence dynamics, pipe flow, and atmospheric and oceanic fluid dynamics.

\section{Dr. Mikhail Goman}

Dr. M. Goman's interests and expertise are in the areas of computational methods for investigation of critical flight regimes (high incidence departure, spin, inertia roll-coupling, flutter, etc.), modeling of unsteady aerodynamics at separated flow conditions (experimental, computational and phenomenological methods), nonlinear control methods for flight envelope prevention and expansion, etc. Dr. M. Goman graduated from the Moscow Institute of Physics and Technology in 1972. Working in the Central Aerohydrodynamic Institute (TsAGI), Russia (1972-2000) and at De Montfort University, UK, since 1997, he gained and contributed his expertise in many research projects in Russia, China, India and the European Union. 


\section{Dr. Yuri Gurevich}

Dr. Y. Gurevich graduated from the Moscow Institute of Physics and Technology with a degree in Fluid Dynamics. Dr. Y. Gurevich spent eighteen years doing research in the aerospace industry, and providing consulting to the nuclear industry and the Soviet Union National Olympic Team. From 1990, Dr. Y. Gurevich led various R\&D programs at the University of Toronto, Canada, and in private and government owned companies in North America. In 2006, Dr. Y. Gurevich formed Research in Flows Inc. His current interests are turbulence dynamics, interaction of turbulence with optical and acoustical instrumentation, and flow analysis in industrial systems.

\section{Dr. Armando Lopez}

Dr. A. Lopez completed his doctorate in Nuclear Physics at McMaster University, Canada, and his Bachelors of Applied Science in Nuclear Engineering at the University of Toronto, Canada. Since 1993, as Chief Engineering Officer of Advanced Measurement \& Analysis Group Inc. (AMAG), Dr. A. Lopez led the development of flow measurement products, and their application in power plants. Prior to this, as Section Head in the Fuel and Physics Department of Ontario Hydro Nuclear, he supported the operation of CANDU reactors in the areas of performance monitoring, data acquisition, non-intrusive flow measurement, fuel management, nuclear plant commissioning, and reactor physics. 


\section{References}

[1] Bradshaw, P., Goodman, D.G. The Effect of Turbulence on StaticPressure Tubes, Ministry of Technology, Aeronautical Research Council Reports and Memoranda No 3527, London: Her Majestys Stationary Office, 1968

[2] Papadopulus, K.H., Stefantos, N.C., Paulsen, U.S., Morfiagakis, E. Effect of Turbulence and Flow Inclination on the Performance of Cup Anemometers in the Field. Boundary-Layer Meteorology, 101 (1), pp77107, Oct. 2001

[3] Berrebi, J., Martinsson, P.-E., Willatzen, M., Delsing, J. Ultrasonic flow metering errors due to pulsating flow. Flow Measurement and Instrumentation, Volume 15, Issues 3, June 2004 pp 179-185.

[4] Weber, F.J., Johari, H., Durgin, W.W. Ultrasonic Beam Propagation in Turbulent Flow. Proceedings of ASME FEDSM'01, 2001, p. 85-91

[5] Strunz, T., Wiest, A., Fleury, A., Frohlich, T. Influence of turbulence on ultrasonic flow measurements. Proc. Of the 5th International Conference on Hydraulic Efficiency Measurements. IGHEM2004. 1-6. (2004)

[6] Jarrin, N., Benhamadouche, S., Laurence, D., Prosser, R. A. Syntheticeddy-method for generating inflow conditions for large-eddy simulations. International Journal of Heat and Fluid Flow 27 (2006) 585593.

[7] Pamis, M., Weiss, P.-., Garnier, E., Deck, S., Sagaut, P. Generation of synthetic turbulent inflow data for large eddy simulation of spatially evolving wall-bounded flows. Physics of Fluids 21, 045103, 2009

[8] Keating, A., Piomelli, U., Balaras, E., Kaltenbach, H.-J. A priori and a posteriori tests of inflow conditions for large-eddy simulation. Physics of Fluids 16 (12), 46964706. 2004.

[9] Lund, T., Wu, X., Squires, D. Generation of turbulent inflow data for spatially-developing boundary layer simulations. Journal of Computational Physics 140, 233258. 1998.

[10] di Mare, L., Klein, M., Jones, W. P., Janicka, J. Synthetic turbulence inflow conditions for large-eddy simulation. Physics of Fluids, 18, 025107, 2006 
[11] Lee, S., Lele, S.K., Moin, P. Simulation of spatially evolving turbulence and the applicability of Taylor's hypothesis in compressible flow. Phys. Fluids A, Vol. 4, No. 7, July 1992

[12] Nicolleau, F.C.G.A., Nowakowski, A.F., Michelitsch, T.M. Synthetic turbulence prediction in non-Kolmogorov turbulence. 13th European Turbulence Conference (ETC13), Journal of Physics: Conference Series 318 (2011) 042009.

[13] Adamian, D., Travin, A. An Efficient Generator of Synthetic Turbulence at RANSLES Interface in Embedded LES of Wall-Bounded and Free Shear Flows, A. Kuzmin (ed.), Computational Fluid Dynamics 2010, DOI 10.1007/978-3-642-17884-9-94, C Springer-Verlag Berlin Heidelberg 2011

[14] Basu, S., Foufoula-Georgiou, E., Port-Agel, F. Synthetic turbulence, fractal interpolation, and large-eddy simulation, Phys. Rev. E 70, 026310 Published 31 August 2004.

[15] Taylor, G.I. The Spectrum of Turbulence. Proceedings of the Royal Society of London. Series A. Mathematics and Physics Science, Vol. 164, No. 919. (Feb. 18, 1938), pp 476-490.

[16] Beck, M.S., Plaskowsky, A. Cross-Correlation Flow Meters, Their Design and Applications. Bristol: Adam Hilger, 1987; ISBN: 085274532X9780852745328. OCLC: 476131509.

[17] Worch, A. A Clamp-on Ultrasonic Cross-Correlation Flow Meter for One Phase Flow, Measurement Science and Technology 9, pp. 622-630 (1998).

[18] Hans, V. Which Physical Quantity of Turbulent Structure is Measured in Cross Correlation Flowmeters? Proceedings, XVII IMEKO World Congress, June 22 27, 2003, Dubrovnik, Croatia 
[19] Gurevich, Y., Lopez, A., Flemons, R., Zobin, D. Theory and Application of Non-Intrusive Ultrasonic Cross-Correlation Flow Meter in Harsh Environment. The 9th International Conference on Flow Measurement, FLOMEKO98.

[20] Lysak, P.D., Jenkins, D.M., Capone, D.E., Brown, W.L. Analytical Model of an Ultrasonic Cross-Correlation Flow Meter, Part I: Stochastic Modeling of Turbulence, Flow Measurement and Instrumentation, 19, (2008) 1-7.

[21] Lysak, P.D., Jenkins, D.M., Capone, D.E., Brown, W.L. Analytical Model of an Ultrasonic Cross-Correlation Flow Meter, Part II: Application, Flow Measurement and Instrumentation, 19 (2008) 41-46.

[22] Frisch, U. Turbulence: the legacy of A.N. Kolmogorov. Cambridge University Press, Cambridge, UK. 1995

[23] Taylor, G.I. Correlation Measurements in a Turbulent Flow Throw a Pipe. Proceedings of the Royal Society of London, Series A, Mathematical and Physical Science, Volume 157, Issue 892, pp 537-546, December 1936. (http://rspa.royalsociietypublishing.org) 